\title{
Can people with osteoarthritis derive clinical benefit from dietary and lifestyle changes? A pilot study
}

\author{
S. Brewer and M. Rayman \\ School of Biomedical and Molecular Sciences, University of Surrey, Guildford GU2 7XH, UK
}

\begin{abstract}
Osteoarthritis is a leading cause of pain and disability in the western world ${ }^{(1)}$. Patient education is the cornerstone of successful management, and patients should ideally receive written information to support their self-care ${ }^{(2)}$. The quality of the written information is of paramount importance, but is often inadequate ${ }^{(3)}$. Booklets typically provide very little nutritional information. This pilot study explored, whether an evidence-based, nutritional booklet could encourage dietary and lifestyle changes and improve symptoms in people with osteoarthritis of the hip and/or knee. Evidence-based approaches were identified from the textbook, Nutrition \& Arthritis ${ }^{(4)}$ and from a search of the published literature. The information was incorporated into a coloured, illustrated 40-page lay booklet entitled Nutrition and Osteoarthritis. Thirty-one men and women with self-reported hip and/or knee osteoarthritis completed a Western Ontario and McMaster Universities Osteoarthritis Index (WOMAC) questionnaire at base-line, and 12 weeks after receiving the booklet. A $20 \%$ relative reduction in symptom scores is considered a clinically important change ${ }^{(5)}$. Analysis of paired data from 21 participants who returned both questionnaires showed mean relative reductions in WOMAC symptom scores of $21.95 \%$ for pain $(95 \% \mathrm{CI} \pm 1.32 ; P=0.026), 21.26 \%$ for stiffness (95\% CI $\pm 0.67 ; P=0.028), 22.47 \%$ for physical function $(95 \% \mathrm{CI} \pm 4.38 ; P=0.012)$ and $22.25 \%$ for global score $(95 \%$ $\mathrm{CI} \pm 5.92 ; P=0.10$ ). Effect sizes were moderate to large (Cohen's $d$ between 0.42 and 0.52 ). This pilot study suggests that an evidencebased booklet that encourages dietary and lifestyle changes can provide clinically meaningful benefit for people with osteoarthritis of the hip and/or knee.
\end{abstract}

1. World Health Organisation (2003) The burden of musculoskeletal conditions at the start of the new millennium: report of a WHO Scientific Group. WHO Technical Report Series No. 919. Geneva: WHO.

2. Leslie M (2000) Knee osteoarthritis management therapies. Pain Manag Nurs 1, 51-57.

3. Grime JG \& Ong BN (2007) Constructing osteoarthritis through discourse - a qualitative analysis of six patient information leaflets on osteoarthritis. BMC Musculoskelet Disord 8, 34.

4. Rayman MP \& Callaghan A (2006) Nutrition and Arthritis. Oxford: Blackwell Publishing Ltd.

5. Tubach F, Ravaud P, Beaton D et al. (2007) Minimal clinically important improvement and patient acceptable symptom state for subjective outcome measures in rheumatic disorders. J Rheumatol 34, 1188-93. 